\title{
Effect of the HDAC inhibitor vorinostat on the osteogenic differentiation of mesenchymal stem cells in vitro and bone formation in vivo
}

\author{
Song $X^{1,2,3}$, Kim DE VEIRMAN ${ }^{2,3}$, Holly EVANS ${ }^{4}$, Gaia Cecilia SANTINI ${ }^{2}$, Isabelle VANDE BROEK ${ }^{3}$, Xavier LELEU ${ }^{5}$, Ann DE \\ BECKER $^{2}$, Ben VAN CAMP ${ }^{3}$, Peter CROUCHER ${ }^{6}$, Karin VANDERKERKEN ${ }^{3}$, Ivan VAN RIET ${ }^{2,3}$ \\ ${ }^{1}$ Department of Lung Cancer Surgery, Lung Cancer Institute, Tianjin Medical University General Hospital, Tianjin 300052, China; ${ }^{2}$ Stem \\ Cell Laboratory-Division Clinical Hematology, Universitair Ziekenhuis Brussel (UZ Brussel), Brussels, Belgium; ${ }^{3}$ Department of Hematol- \\ ogy and Immunology-Vrije Universiteit Brussel (VUB), Myeloma Center Brussels, Brussels, Belgium; ${ }^{4}$ Department of Human Metabo- \\ lism, University of Sheffield Medical School, Sheffield, UK; ${ }^{5}$ Service d'Hématologie, Centre Hospitalier Universitaire (CHU), Lille, France; \\ ${ }^{6}$ Garvan Institute for Medical Research, Sydney, Australia
}

Aim: Vorinostat, a histone deacetylase (HDAC) inhibitor currently in a clinical phase III trial for multiple myeloma (MM) patients, has been reported to cause bone loss. The purpose of this study was to test whether, and to what extent, vorinostat influences the osteogenic differentiation of mesenchymal stem cells (MSCs) in vitro and bone formation in vivo.

Methods: Bone marrow-derived MSCs were prepared from both normal donors and MM patients. The MSCs were cultured in an osteogenic differentiation induction medium to induce osteogenic differentiation, which was evaluated by alkaline phosphatase (ALP) staining, Alizarin Red S staining and the mRNA expression of osteogenic markers. Naïve mice were administered vorinostat (100 mg/kg, ip) every other day for 3 weeks. After the mice were sacrificed, bone formation was assessed based on serum osteocalcin level and histomorphometric analysis.

Results: Vorinostat inhibited the viability of hMSCs in a concentration-dependent manner (the IC 50 value was $15.57 \mu \mathrm{mol} / \mathrm{L}$ ). The low concentration of vorinostat $(1 \mu \mathrm{mol} / \mathrm{L})$ did not significantly increase apoptosis in hMSCs, whereas pronounced apoptosis was observed following exposure to higher concentrations of vorinostat (10 and $50 \mu \mathrm{mol} / \mathrm{L}$ ). In bone marrow-derived hMSCs from both normal donors and MM patients, vorinostat $(1 \mu \mathrm{mol} / \mathrm{L})$ significantly increased ALP activity, mRNA expression of osteogenic markers, and matrix mineralization. These effects were associated with upregulation of the bone-specifying transcription factor Runx 2 and with the epigenetic alterations during normal hMSCs osteogenic differentiation. Importantly, the mice treated with vorinostat did not show any bone loss in response to the optimized treatment regimen.

Conclusion: Vorinostat, known as a potent anti-myeloma drug, stimulates MSC osteogenesis in vitro. With the optimized treatment regimen, any decrease in bone formation was not observed in vivo.

Keywords: histone deacetylase (HDAC); vorinostat; mesenchymal stem cell; osteogenesis; alkaline phosphatase (ALP); Runx2; osteocalcin; multiple myeloma

Acta Pharmacologica Sinica (2013) 34: 699-709; doi: 10.1038/aps.2012.182; published online 8 Apr 2013

\section{Introduction}

Multiple myeloma (MM) is a malignant plasma cell (PC) disorder characterized by an accumulation of monoclonal, terminally differentiated PCs in the bone marrow (BM). Approximately $80 \%$ of patients with newly diagnosed MM show evidence of bone disease by conventional radiography ${ }^{[1]}$.

\footnotetext{
* To whom correspondence should be addressed.

E-mail ivan.vanriet@uzbrussel.be

Received 2012-08-21 Accepted 2012-12-05
}

The development of myeloma bone disease is caused by an uncoupling of normal bone remodeling, which is the result of increased osteoclast activity and markedly suppressed osteoblast function ${ }^{[2,3]}$.

Mesenchymal stem cells (MSCs) are mostly present in the $\mathrm{BM}$ and are characterized by their ability to give rise to mesodermal cell types, such as osteoblasts, adipocytes and chondrocytes $^{[4]}$. In addition to their multi-lineage differentiation potential, MSCs also possess multiple biological properties, including the production of a variety of cytokines/ 
chemokines, immunomodulary properties and the ability to migrate towards sites of injury or tumor location. These features make them useful for a number of potential therapeutic applications, including supporting hematopoiesis, treating graft-versus-host disease (GVHD), tissue engineering and gene therapy ${ }^{[5-8]}$.

MSCs from myeloma patients (MM-hMSCs) show an enhanced cytokine production and a distinctive gene expression profile compared to hMSCs from normal donors (NDhMSCs $)^{[9,10]}$. Moreover, we previously observed that MMhMSCs, especially those from MM patients who exhibit bone lesions, showed impaired differentiation towards osteoblasts compared to ND-hMSCs ${ }^{[11]}$. The suppression of MSC osteogenic differentiation is due to the presence of soluble factors produced by MM cells and the abnormal regulation of signaling pathways in $\mathrm{MSCs}^{[11-16]}$.

The strategy of targeting endogenous MSCs for committed differentiation in vivo using pharmacological agents has recently been emphasized. Bortezomib is a clinically available proteasome inhibitor that is used for the treatment of MM. Preclinical and clinical observations have revealed that bortezomib is not only a potent anti-MM drug but is also capable of promoting bone formation ${ }^{[17,18]}$. Therefore, it would be interesting to investigate how other anti-myeloma drugs regulate bone formation. Histone deacetylase inhibitors (HDACi) are considered one of the most promising drugs for the treatment of neoplastic disorders. Although these drugs are primarily studied for their anti-proliferative and pro-apoptotic activities against tumors, there is growing evidence that some HDACi also stimulate osteogenic differentiation of $\mathrm{MSCs}^{[19-23]}$. As a pan-inhibitor of class I and II HDAC proteins, vorinostat (SAHA or Zolinza ${ }^{\mathrm{TM}}$ ) is one of the HDACi currently being used to treat MM patients. Vorinostat, alone and in combination with bortezomib, are both being evaluated in clinical phase I/ II trials in $\mathrm{MM}^{[24,25]}$, while the combination of vorinostat and lenalidomide is currently being tested in a phase III clinical trial ${ }^{[26]}$. However, previous publications showed that vorinostat decreased MSC osteogenic differentiation ability in vitro and caused bone loss in vivo ${ }^{[27,28]}$. It is unclear why vorinostat apparently induces the opposite effect on MSC osteogenic differentiation in vitro compared to other HDACi. Moreover, it is important to explore whether the bone loss promoting effect of vorinostat could be blunted or reversed by adjusting the treatment strategy in vivo.

\section{Materials and methods Reagents}

Vorinostat was kindly provided by Merck \& Co Inc (Whitehouse station, NJ, USA). It was dissolved in DMSO as a 20 $\mathrm{mmol} / \mathrm{L}$ stock solution and diluted to the required concentrations.

\section{Primary culture of human MSCs}

BM samples from healthy donors and MM patients were obtained after informed consent, in accordance with the Declaration of Helsinki. BM aspirates were obtained from the sternum of healthy donors or from the iliac crest of myeloma patients. For hMSCs from healthy donors, whole BM mononuclear cells were collected by density gradient centrifugation with Ficoll-Hypaque (Nycomed, Takeda, Brussels, Belgium) and cultured according to a previously described method ${ }^{[29]}$. For hMSCs from MM patients, an additional CD138 MACS separation was performed to remove the malignant plasma cells before the initial culture. hMSCs were used at passage 3-5 in this study. Prior to their use in these experiments, hMSCs were characterized by their immunophenotype $\left(\mathrm{CD} 90^{+}, \mathrm{CD}^{+}, \mathrm{CD}^{+} 66^{+}, \mathrm{CD} 105^{+}\right.$and $\left.\mathrm{CD} 45^{-}\right)$(Figure 1). This study was approved by the local ethical committee.

\section{Human myeloma cell lines}

Three well-characterized human MM cell lines (Karpas 707, RPMI 8226 and U266) were used in our experiments. They were maintained in RPMI-1640 medium containing 10\% FCS, supplemented with $1 \% \mathrm{~L}$-glutamine and $1 \%$ penicillin/streptomycin (Life Technologies, Gent, Belgium) at $37^{\circ} \mathrm{C}$ in $5 \% \mathrm{CO}_{2}$.

\section{Viability assays}

hMSCs and MM cells were cultured at a density of 2000 cells/ well in a 96-well plate in the complete growth medium with various concentrations of vorinostat $(0 \mathrm{nmol} / \mathrm{L}, 10 \mathrm{nmol} / \mathrm{L}$, $100 \mathrm{nmol} / \mathrm{L}, 500 \mathrm{nmol} / \mathrm{L}, 1 \mu \mathrm{mol} / \mathrm{L}, 5 \mu \mathrm{mol} / \mathrm{L}, 10 \mu \mathrm{mol} / \mathrm{L}$, and $50 \mu \mathrm{mol} / \mathrm{L}$ ). After $72 \mathrm{~h}$, cell viability was measured using CellTiter-Glo ${ }^{\circledR}$ (Promega, Madison, WI, USA), according to the manufacturer's protocol.

\section{Cell cycle analysis}

Three hundred thousand hMSCs cells were cultured in complete growth medium containing different concentrations of vorinostat. After $72 \mathrm{~h}$ incubation, the cells were collected in FACS tubes and washed once in cold PBS. Next, they were
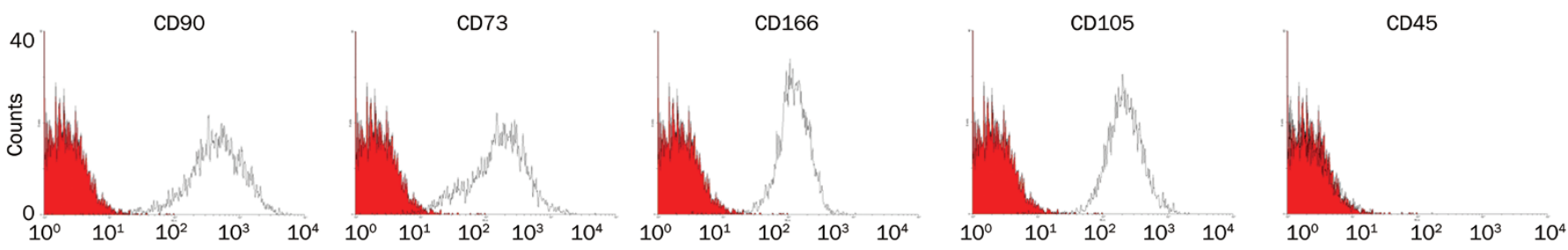

Figure 1. Immunophenotype of hMSCs. hMSCs from normal donors and MM patients were positive for CD90, CD73, CD166, CD105, and negative for CD45. Representative FACS staining of one ND-hMSCs is shown. 
resuspended in $500 \mu \mathrm{L}$ staining solution containing $50 \mu \mathrm{g} / \mathrm{mL}$ propidium iodide (PI) (BD Pharmingen, Franklin Lakes, NJ, USA), $0.1 \%(v / v)$ Triton $\mathrm{X}-100$, and $0.1 \%$ (wt/vol) sodium citrate. Cells were incubated at $4^{\circ} \mathrm{C}$ in the dark for $15 \mathrm{~min}$ and analyzed with a FACSCanto flow cytometer (BD Pharmingen), using FACS Diva software.

\section{Apoptosis analysis}

After culture in complete growth medium with different concentrations of vorinostat for $72 \mathrm{~h}, 2 \times 10^{5}$ MSCs were washed twice with PBS and stained with $3 \mu \mathrm{L}$ 7-AAD and $4 \mu \mathrm{L}$ Annexin V-FITC (BD Pharmingen) in $100 \mu \mathrm{L}$ buffer. The cells were then incubated at $4{ }^{\circ} \mathrm{C}$ for $15 \mathrm{~min}$. The cells were resuspended in $400 \mu \mathrm{L}$ of binding buffer and immediately analyzed using a FACSCanto flow cytometer (BD Pharmingen), using FACS Diva software.

\section{Western blot analysis}

Preparation of whole cell lysates and immunoblotting were performed as previously described ${ }^{[30]}$, using the following antibodies: acetyl-histone 3 (Ac-H3) (Upstate, Lake Placid, NY, USA), p21 (Santa Cruz, Santa Cruz, CA, USA), $\beta$-actin, HDAC1 and HDAC4 (Cell Signaling Technology, Danvers, MA, USA). Horseradish peroxidase-conjugated secondary antibodies were purchased from Amersham (Buckinghamshire, UK). Band intensities were quantified using NIH ImageJ software (http://rsbweb.nih.gov/ij/), expressed in arbitrary units of optical density and normalized to the intensity of $\beta$-actin in the same blot.

\section{RNA extraction and cDNA synthesis}

Total RNA was extracted using Trizol reagent (Invitrogen) and the RNeasy mini kit (Qiagen, Venlo, the Netherlands), and cDNA was synthesized using the Thermo Scientific Verso $^{\mathrm{TM}}$ cDNA synthesis kit (Thermo Scientific, Ulm, Germany), according to the manufacturer's protocol.

\section{Quantitative real-time PCR}

Runx2 mRNA expression was quantified using the SYBR GreenER ${ }^{\mathrm{TM}}$ qPCR for iCycler kit (Life Technologies), using an iCycler Thermal Cycler (Bio-Rad, Eke, Belgium). Transcript levels were normalized to the housekeeping gene, $\beta$-actin, and analyzed using the relative quantification $2^{-\Delta \Delta \mathrm{Ct}}$ method. Primer sequences are reported in Table 1.

\section{RT-PCR}

The expression of osteogenic markers was determined by RT-PCR, as described previously ${ }^{[28]}$. Primer sequences are reported in Table 2 .

\section{Osteogenic differentiation induction}

Twenty thousand hMSCs were plated in $1.5 \mathrm{~mL}$ growth medium in a 12-well plate. After overnight incubation, osteogenic differentiation was induced with Osteogenesis Induction Medium (OIM) (Lonza, Verviers, Belgium) (containing dexamethasone, ascorbate, glycerophosphate, L-glutamine,
Table 1. Real-time PCR primers (human).

\begin{tabular}{lccc}
\hline Gene & Primer & $\begin{array}{l}\text { GenBank } \\
\text { accession }\end{array}$ & $\begin{array}{c}\text { Annealing } \\
\text { temp }\left({ }^{\circ} \mathrm{C}\right)\end{array}$ \\
\hline Runx2 & $\begin{array}{l}\text { 5'-GGAGTGGACGAGGCAAGAGTTT-3' } \\
\text { 5'-AGCTTCTGTCTGTGCCTTCTGG-3' }\end{array}$ & NM_009820 & 60 \\
B-Actin & 5'-ATGTGGCCGAGGACTTTGATT-3' & NM_001101 & 60 \\
& 5'-AGTGGGGTGGCTTTTAGGATG-3' & & \\
\hline
\end{tabular}

Runx2, runt-related transcription factor 2 .

Table 2. RT-PCR primers (human).

\begin{tabular}{|c|c|c|c|}
\hline Gene & Primer & $\begin{array}{l}\text { GenBank } \\
\text { accession }\end{array}$ & $\begin{array}{l}\text { Annealing } \\
\text { temp }\left({ }^{\circ} \mathrm{C}\right)\end{array}$ \\
\hline ALP & $\begin{array}{l}\text { 5'-TGGAGCTTCAGAAGCTCAACACCA-3' } \\
\text { 5'-ATCTCGTTGTCTGAGTACCAGTCC-3' }\end{array}$ & NG_008940 & 51 \\
\hline OPN & $\begin{array}{l}\text { 5'-ACGCCGACCAAGGAAAACTC-3' } \\
\text { 5'-GTCCATAAACCACACTATCACCTCG-3' }\end{array}$ & NM_000582 & 51 \\
\hline BSP & $\begin{array}{l}\text { 5'-AATGAAAACGAAGAAAGCGAAG-3' } \\
\text { 5'-ATCATAGCCATCGTAGCCTTGT-3' }\end{array}$ & NM_004967 & 51 \\
\hline BMP2 & $\begin{array}{l}\text { 5'-CCACCATGAAGAATCTTTGG-3' } \\
\text { 5'-CCACGTACAAAGGGTGTCTC-3' }\end{array}$ & NM_001200 & 55 \\
\hline$\beta$-Actin & $\begin{array}{l}\text { 5'-ATGTGGCCGAGGACTTTGATT-3' } \\
\text { 5'-AGTGGGGTGGCTTTTAGGATG-3' }\end{array}$ & NM_001101 & 60 \\
\hline
\end{tabular}

ALP, alkaline phosphatase; OPN, osteopontin; BSP, bone sialoprotein; BMP2, bone morphogenetic protein 2 .

Pen/Strep and mesenchymal cell growth supplement), with or without vorinostat. The medium was replaced every three days. ALP expression and calcium deposit were used as early and late markers for osteogenesis, respectively, and were detected by ALP and Alizarin Red S staining, respectively.

\section{Qualitative ALP staining}

After culture in osteogenic induction medium with or without vorinostat $(1 \mu \mathrm{mol} / \mathrm{L})$ for the indicated time, cells were washed with PBS, and BCIP/NBT (5-bromo-4-chloro-3-indolyl phosphate/Nitroblue tetrazolium, Sigma-Aldrich, Bornem, Belgium) liquid substrate was added to each well, followed by a 60 min incubation in the dark. ALP positive cells were visualized under a light microscope (dark purple color).

\section{Quantitative ALP activity analysis}

To quantify ALP activity, $2 \times 10^{3} \mathrm{hMSC}$ s were cultured in osteogenic induction medium in a 96-well plate with or without vorinostat $(0 \mathrm{nmol} / \mathrm{L}, 100 \mathrm{nmol} / \mathrm{L}, 500 \mathrm{nmol} / \mathrm{L}, 1 \mu \mathrm{mol} / \mathrm{L}$, $5 \mu \mathrm{mol} / \mathrm{L}$, and $10 \mu \mathrm{mol} / \mathrm{L})$. Three days later, the cells were washed once with PBS, and $100 \mu \mathrm{L}$ Alkaline Phosphatase Yellow (pNPP) Liquid ELISA substrate (Sigma-Aldrich) was added for $30 \mathrm{~min}$ in the dark. Finally, ALP activity was analyzed using an ELISA reader at $415 \mathrm{~nm}$. ALP activity was normalized to the amount of total protein in each well to determine the ALP index. 


\section{Alizarin Red S staining}

After two weeks culture in osteogenic induction medium, with or without vorinostat $(1 \mu \mathrm{mol} / \mathrm{L})$, cells were washed with PBS and fixed with $10 \%$ paraformaldehyde (Merck) for 15 min at room temperature. After washing, the cells were stained with $40 \mathrm{mmol} / \mathrm{L}$ fresh Alizarin Red solution ( $\mathrm{pH}=4.2)$ and incubated for $10 \mathrm{~min}$ at room temperature with gentle shaking. Then, the Alizarin Red solution was aspirated and the wells were washed at least three times before observation. Calcium deposits were visualized by their red color. To quantify the staining, cultures were distained using 10\% cetylpyridinium chloride (CPC) in $10 \mathrm{mmol} / \mathrm{L}$ sodium phosphate $(\mathrm{pH}=7.0)$ for $15 \mathrm{~min}$ at room temperature. Aliquots of the exacts were diluted 10-fold in 10\% CPC solution, and the concentration of Alizarin Red S was determined by measuring absorbance at $562 \mathrm{nmol} / \mathrm{L}$ with a multiplate reader (Thermo Labsystems, VWR International, Leuven, Belgium).

\section{In vivo study}

C57BL/KaLwRij mice were purchased from Harlan (Horst, the Netherlands), and experiments were performed when they were 6 to 8 weeks of age. The mice were housed and maintained following the conditions approved by the Ethical Committee for Animal Experiments, Vrije Universiteit Brussels (license No LA1230281). The animal ethics meet the standards required by the United Kingdom Coordinating Committee on Cancer Research Guidelines (UKCCCR, 1998).

McGee-Lawrence et al and Pratap et al reported that treatment with vorinostat $(100 \mathrm{mg} / \mathrm{kg})$ daily for 3 or 4 weeks caused bone loss in naive mice ${ }^{[27,28]}$. However, a previous study by Campbell et al showed that intraperitoneal (ip) administration of vorinostat $(100 \mathrm{mg} / \mathrm{kg})$ for $2 \mathrm{~d}$ per week produced a marked decrease in MM tumor burden in vivo, and the anti-MM effect did not increase when the frequency of drug treatment increased to $5 \mathrm{~d}$ per week ${ }^{[31]}$. Therefore, we exploited a less frequent treatment strategy in our study. C57BL/KaLwRij mice ( $n=5$ per group) were injected ip with either vehicle (DMSO) or vorinostat $(100 \mathrm{mg} / \mathrm{kg})$, and treatments were administered every other day for three weeks (three times/week). The animals were weighed every week and were sacrificed after $21 \mathrm{~d}$.

\section{Serum osteocalcin measurement}

Peripheral blood was collected at the end of the experiment, and the mouse serum was diluted 1/20. Osteocalcin levels were assessed by ELISA (Demeditec Diagnostics, Kiel, Germany) according to the manufacturer's instructions.

\section{Colony formation assay}

$\mathrm{BM}$ was flushed from the femur and tibiae into McCoy's 5A medium containing 1\% L-glutamine (2 mmol/L), $100 \mathrm{U} / \mathrm{mL}$ penicillin/streptomycin and $20 \%$ stem cell mouse growth supplement (StemCell Technologies, Vancouver, BC, Canada). After red blood cell lysis, $1 \times 10^{6}$ primary BM cells from each mouse were plated in 12-well plates, and the medium was changed at $24 \mathrm{~h}$ to eliminate non-adherent cells. To examine the osteogenic differentiation potential of primary murine MSCs (CFU-Alp), cells were moved to OIM (Lonza) at d 3, when primary colonies formed. Ten days later, ALP staining was carried out with BCIP/NBT solution (Sigma-Aldrich) per the manufacturer's instructions. Only colonies containing a majority of stained cells ( $>50 \%$ ) were scored as positive.

\section{Histomorphometric analysis}

The tibiae were decalcified and embedded in paraffin, and $3-\mu \mathrm{m}$ sections were cut and stained with H\&E or enzymatically stained for tartrate resistant acid phosphatase. Osteoblasts were identified on the basis of characteristic morphology, and osteoclasts were identified following tartrate resistant acid phosphatase staining. The number of osteoclasts and osteoblasts per millimeter were measured using Osteomeasure bone histomorphometry software (OsteoMetrics, Decatur, GA, USA).

\section{Statistical analysis}

Statistical analysis was performed using GraphPad Prism 5 software. All data represent the mean $\pm S D$, and results were analyzed using the Mann Whitney test (for two groups) and one way ANOVA, followed by Tukey's post test (for more than two groups). $P<0.05$ was considered statistically significant. All experiments were repeated at least three times.

\section{Results}

\section{Effect of vorinostat on hMSC growth in vitro}

Vorinostat reduced cell growth in a concentration-dependent manner in MM cells (RPMI8226, Karpas 707, and U266) and hMSCs. Importantly, we observed that vorinostat affected the viability of hMSCs with an $\mathrm{IC}_{50}$ (half maximal inhibitory concentration) of $15.57 \mu \mathrm{mol} / \mathrm{L}$, while the observed $\mathrm{IC}_{50} \mathrm{~s}$ for the MM cell lines, RPMI8226, Karpas 707, and U266, were 0.71 $\mu \mathrm{mol} / \mathrm{L}, 0.24 \mu \mathrm{mol} / \mathrm{L}$, and $1.41 \mu \mathrm{mol} / \mathrm{L}$, respectively (Figure 2A).

To understand the underlying mechanism by which vorinostat inhibited hMSC growth, cell cycle distribution was assessed by PI staining. At concentrations of 1 and $10 \mu \mathrm{mol} / \mathrm{L}$, vorinostat caused significant $\mathrm{G}_{2} / \mathrm{M}$ phase arrest compared to untreated cells. An increase in the apoptotic population (sub- $\mathrm{G}_{0} / \mathrm{G}_{1}$ ) was observed as well, following treatment with $10 \mu \mathrm{mol} / \mathrm{L}$ vorinostat (Figure $2 \mathrm{~B}$ ).

Annexin V/7AAD staining revealed that vorinostat (1 $\mu \mathrm{mol} / \mathrm{L}$ ) did not significantly increase apoptosis in hMSCs, while more apoptotic cells (early+late apoptosis) were detected after treatment with $5 \mu \mathrm{mol} / \mathrm{L}$ vorinostat. More pronounced apoptosis was observed following exposure to $10 \mu \mathrm{mol} / \mathrm{L}$ and $50 \mu \mathrm{mol} / \mathrm{L}$ vorinostat (Figure 2C).

In addition, we observed that vorinostat induced morphological changes in hMSCs. Under untreated conditions, hMSCs appeared to be elongated and fibroblast-like. When exposed to vorinostat for $24 \mathrm{~h}$, the cells became flatter and more stress fibers could be observed in the cytoplasm (Figure 2D). This phenotype became more prominent with increasing concentrations of vorinostat. 
A
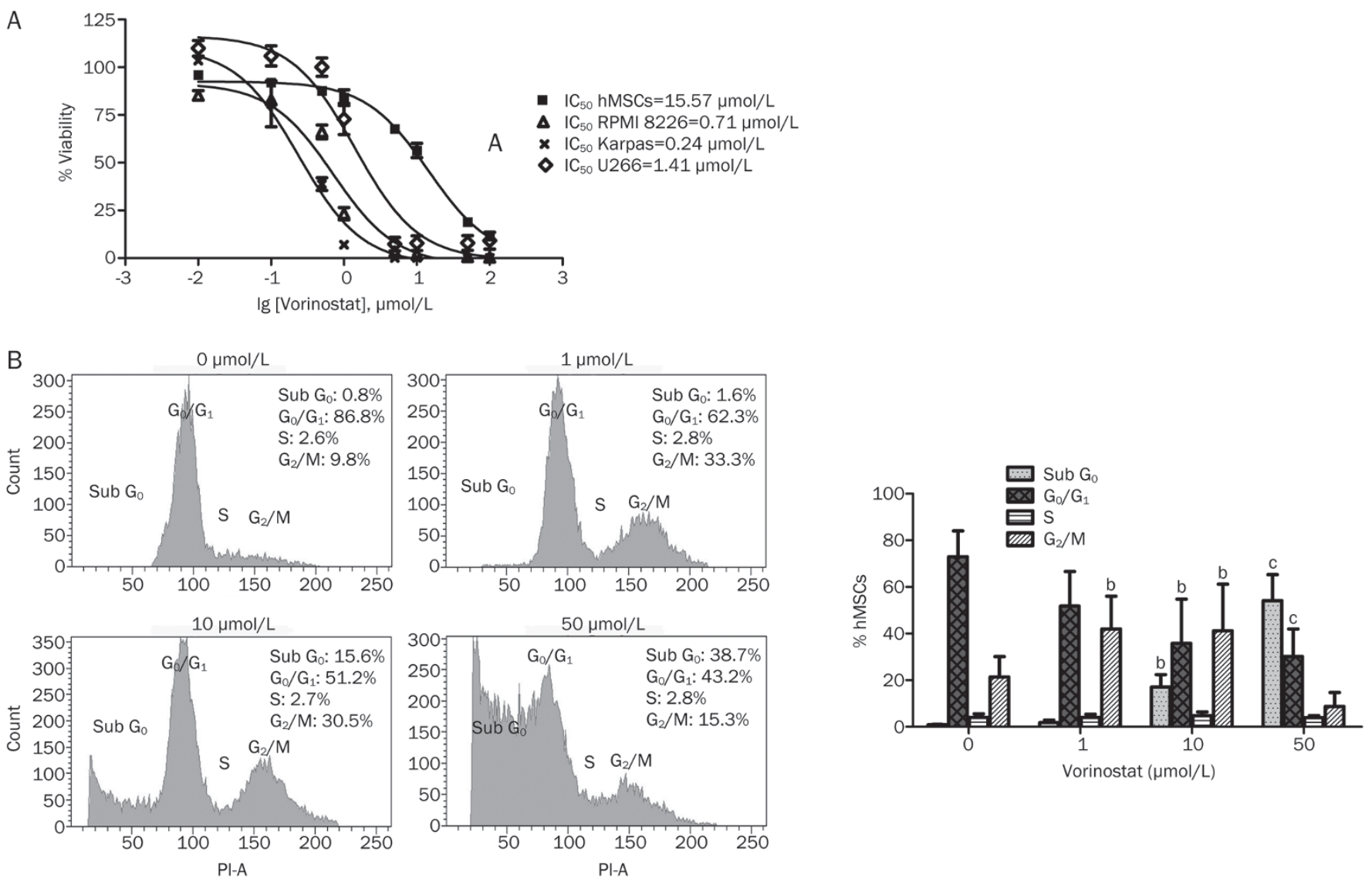

C
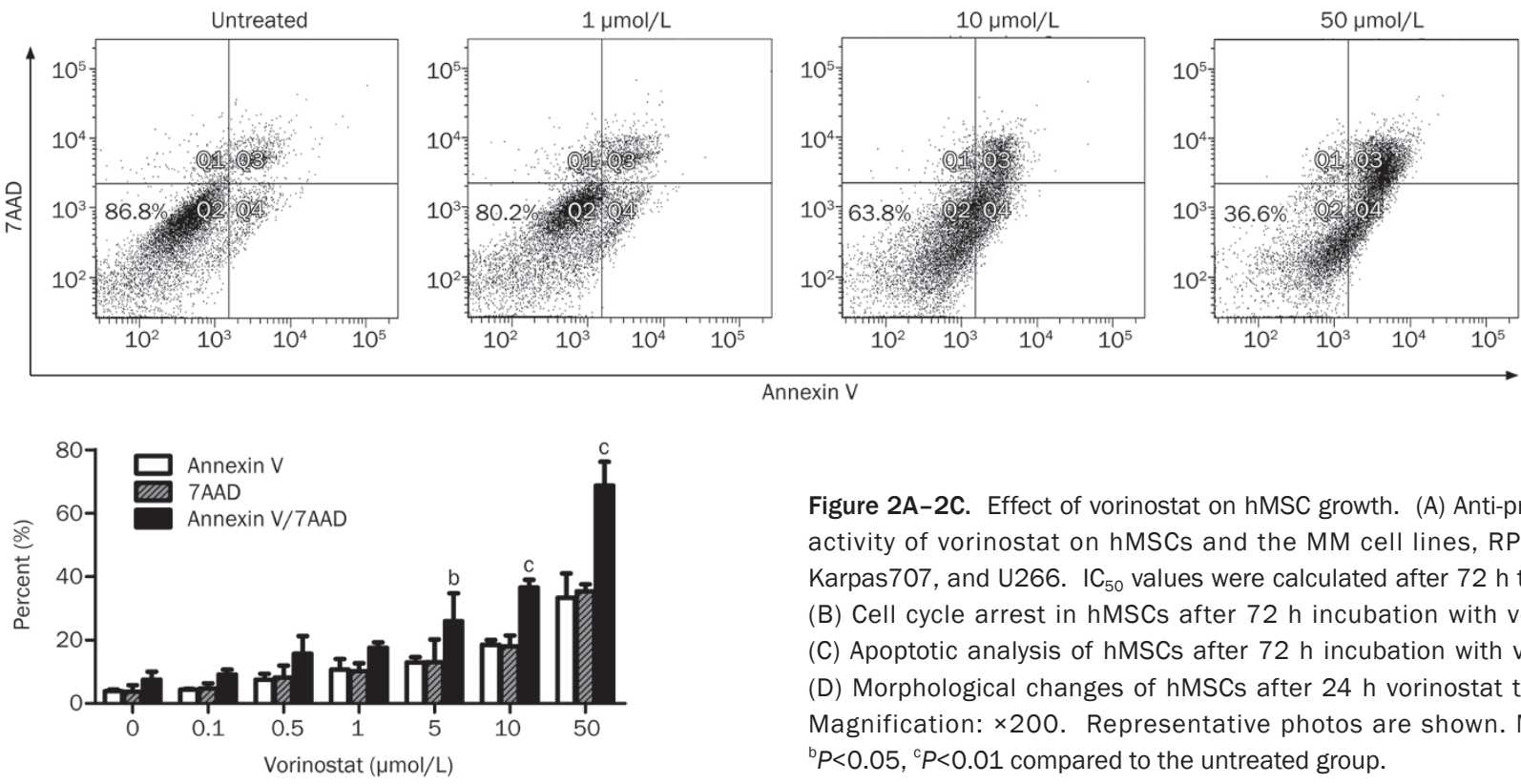

Figure 2A-2C. Effect of vorinostat on hMSC growth. (A) Anti-proliferative activity of vorinostat on hMSCs and the MM cell lines, RPMI 8226, Karpas707, and U266. IC 50 values were calculated after $72 \mathrm{~h}$ treatment. (B) Cell cycle arrest in hMSCs after $72 \mathrm{~h}$ incubation with vorinostat. (C) Apoptotic analysis of hMSCs after $72 \mathrm{~h}$ incubation with vorinostat. (D) Morphological changes of hMSCs after $24 \mathrm{~h}$ vorinostat treatment. Magnification: $\times 200$. Representative photos are shown. Mean \pm SD ${ }^{b} P<0.05,{ }^{c} P<0.01$ compared to the untreated group.

Osteogenesis-promoting effect of vorinostat on BM derived hMSCs from normal donors and MM patients

BM derived ND-hMSCs were cultured in osteogenic medium with increasing concentrations of vorinostat. After $72 \mathrm{~h}$, ALP activity, which is an early marker of osteoblast differentiation, was quantified by ELISA. As demonstrated in Figure 3A, vorinostat significantly increased ALP activity at concentrations of $1 \mu \mathrm{mol} / \mathrm{L}$ and $5 \mu \mathrm{mol} / \mathrm{L}$ compared to the control group, but ALP activity decreased with increasing concentrations of vorinostat due to drug toxicity $(P<0.05)$.

As the $1 \mu \mathrm{mol} / \mathrm{L}$ dose of vorinostat induced the highest relative ALP activity, we further confirmed its effect on hMSC osteogenic differentiation using this concentration. PCR analysis revealed that the expression of OPN, ALP, BSP, and BMP2 

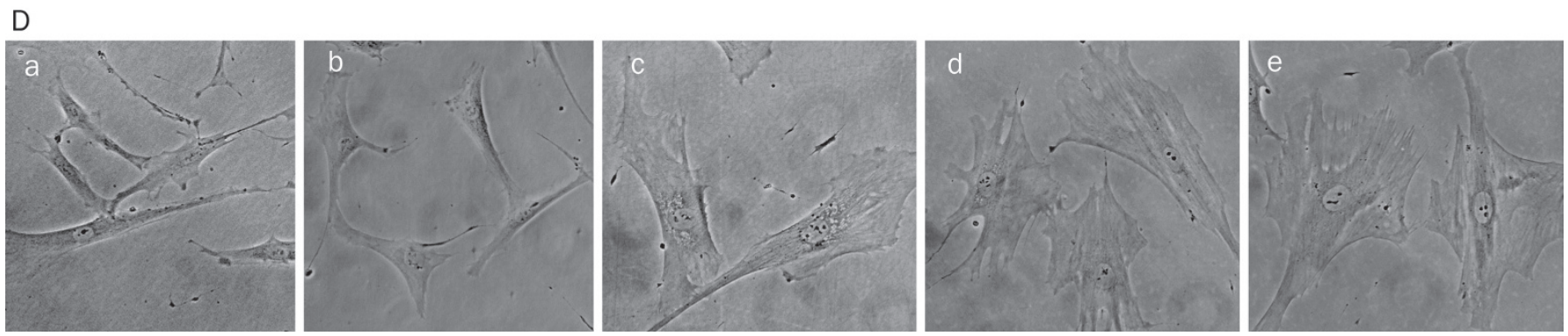

Figure 2D. Morphological changes of hMSCs after $24 \mathrm{~h}$ vorinostat treatment. (a) Untreated cells; (b) vorinostat $0.1 \mu \mathrm{mol} / \mathrm{L}$; (c) vorinostat $0.5 \mu \mathrm{mol} / \mathrm{L}$; (d) vorinostat $1 \mu \mathrm{mol} / \mathrm{L}$; (e) vorinostat $5 \mu \mathrm{mol} / \mathrm{L}$. Magnification: $\times 200$. Representative photos are shown.

increased in vorinostat treated ND-hMSCs (Figure 3B). ALP staining confirmed that more ALP positive cells were present after vorinostat treatment for $7 \mathrm{~d}$ compared to the control (Figure 3C). Finally, Alizarin Red S staining confirmed that Vorinostat increased matrix mineralization in ND-hMSCs (Figure 3D).

We also tested the effect of vorinostat on MM-hMSCs osteogenic differentiation. Our study indicated that MM-hMSCs have impaired osteogenic differentiation compared to their normal counterparts. More importantly, we found that similar to ND-hMSCs, vorinostat was able to increase the activity of ALP (Figure 3A and 3C), the mRNA expression of osteogenic markers (Figure 3B), and calcium deposition (Figure 3D) in MM-hMSCs.

\section{Mechanisms involved in the vorinostat-induced osteogenesis}

hMSCs were cultured in growth medium for $48 \mathrm{~h}$ with or without vorinostat. In vorinostat treated hMSCs, we observed an upregulation of Runx2 expression, which is a key transcription factor for osteogenesis, by real-time PCR (Figure 4A).

In addition, in response to exogenous osteogenic stimuli, hMSCs exhibited a progressive hyperacetylation of histone 3 but suppressed HDAC1 in a time-dependent manner (Figure 4B). Interestingly, without any osteogenic stimuli, vorinostat inhibited HDAC activity and increased the acetylation of histone 3 in hMSCs. This mimics the natural epigenetic alteration of hMSCs during osteogenic differentiation (Figure 4C). We also found that p21, an important marker for the initiation and regulation of osteoblast differentiation ${ }^{[23]}$, increased significantly and in a concentration-dependent manner in hMSCs (Figure 4C).

\section{The effect of vorinostat on bone formation in naive mice}

Naive mice ( $n=5$ per group) received ip injection of either vehicle (DMSO) or vorinostat (100 mg/ $\mathrm{kg}$ ) for 3 weeks (9 dosages) (Figure 5A). As shown in Figure 5B, there was no significant effect on the body weight of mice treated with vorinostat (100 $\mathrm{mg} / \mathrm{kg}$ ) compared to the control group. Importantly, with the adjusted treatment strategy, vorinostat did not cause any bone loss in vivo, contrary to what has been reported in previous publications ${ }^{[27,28]}$. Alternatively, we observed that vorinostat treatment of naive mice (C57BL/KaLwRij) increase the number of osteogenic colonies by 1.32-fold (Figure 6A), increased serum osteocalcin levels by $46.50 \%$ (Figure 6B), and increased osteoblast numbers in endocordical and trabecular bone surfaces by $6.74 \%$ and $19.53 \%$, respectively (Figure 6C); however, the differences in increased osteogenic colonies, serum osteocalcin levels and osteoblast numbers were not significant.

\section{Discussion}

Mammalian cells express 18 HDACs, which are divided into four classes based on sequence conservation and functional similarities ${ }^{[32]}$. The crucial roles of several HDACs, including HDAC1, HDAC3, HDAC4, HDAC5, HDAC6, HDAC8, and Sirt1, in the regulation of bone formation have been identified ${ }^{[23,33-37]}$.

Several natural and synthetic compounds aimed at suppressing HDAC activity have been developed. HDACi act by binding to the catalytic site of $\mathrm{HDACs}^{[38]}$. Vorinostat is a potent pan-HDAC inhibitor that was approved by US FDA for the treatment of cutaneous T cell lymphoma in 2006. Vorinostat has also been used for the treatment of different tumor types ${ }^{[39,40]}$ and has been tested in phase III clinical trials for treatment of MM patients ${ }^{[24-26]}$. In the present study, we observed that while vorinostat was able to inhibit the growth of hMSCs in a concentration-dependent manner, the $\mathrm{IC}_{50}$ for hMSCs is much higher compared to MM cells. Unlike the findings from Di Bernardo et $a l^{[41]}$, we found that vorinostat (1 $\mu \mathrm{mol} / \mathrm{L}$ ) did not induce significant apoptosis and $\mathrm{G}_{0} / \mathrm{G}_{1}$ arrest in hMSCs. In fact, we found that vorinostat induced $\mathrm{G}_{2} /$ $\mathrm{M}$ arrest, which is consistent with other HDAC inhibitors ${ }^{[19]}$. One recent paper suggested that HDACi could induce cell $\mathrm{G}_{2} / \mathrm{M}$ arrest via the p53 pathway in colorectal cancer cells, an observation that deserves further investigation in $\mathrm{MSCs}^{[42]}$. Noticeably, $1 \mu \mathrm{mol} / \mathrm{L}$ vorinostat caused significant MM cell growth inhibition but produced only minor effects on hMSCs. At this concentration, in vitro osteogenic differentiation was stimulated in both normal donors and, more importantly, MM patients. One possible mechanism responsible for the osteogenesis-promoting effect of vorinostat, which is in line with other HDACi ${ }^{[19-21]}$, is that vorinostat can upregulate the transcriptional activation of Runx2, a very important transcription factor for osteoblast differentiation. Moreover, another potential mechanism by which vorinostat promotes osteogenesis might be related to the acceleration of epigenetic modification. We found that during natural hMSCs osteogenic differentia- 
A

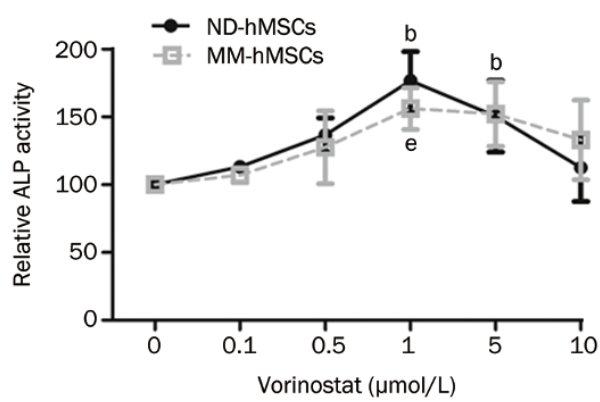

C
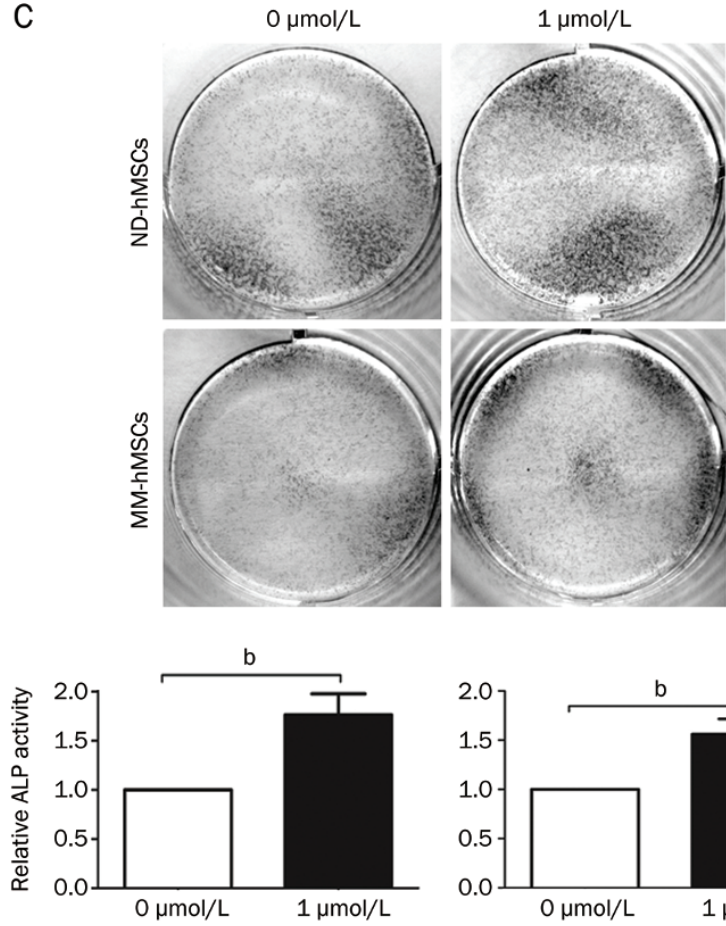

B

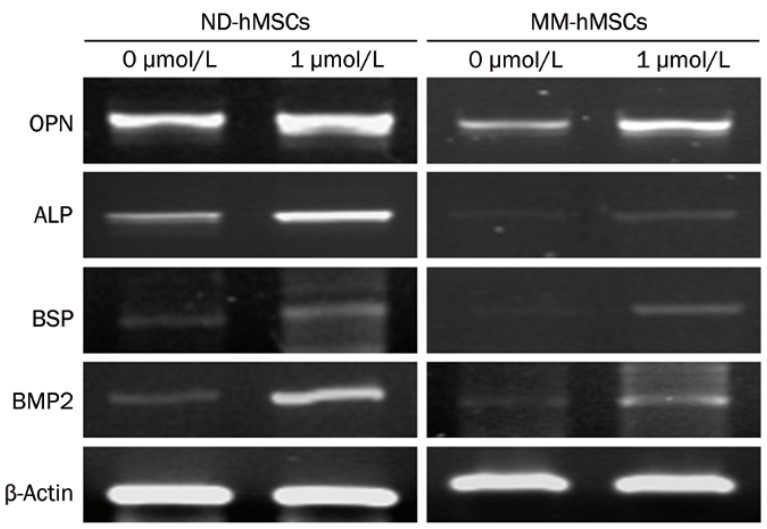

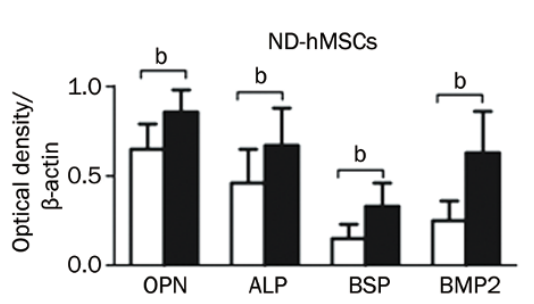

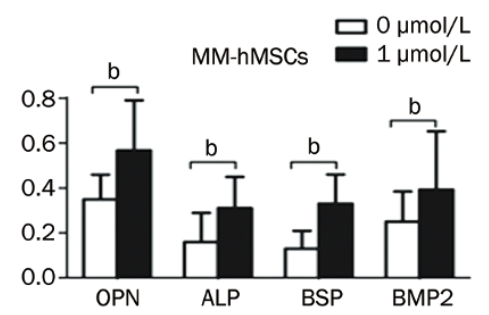

D
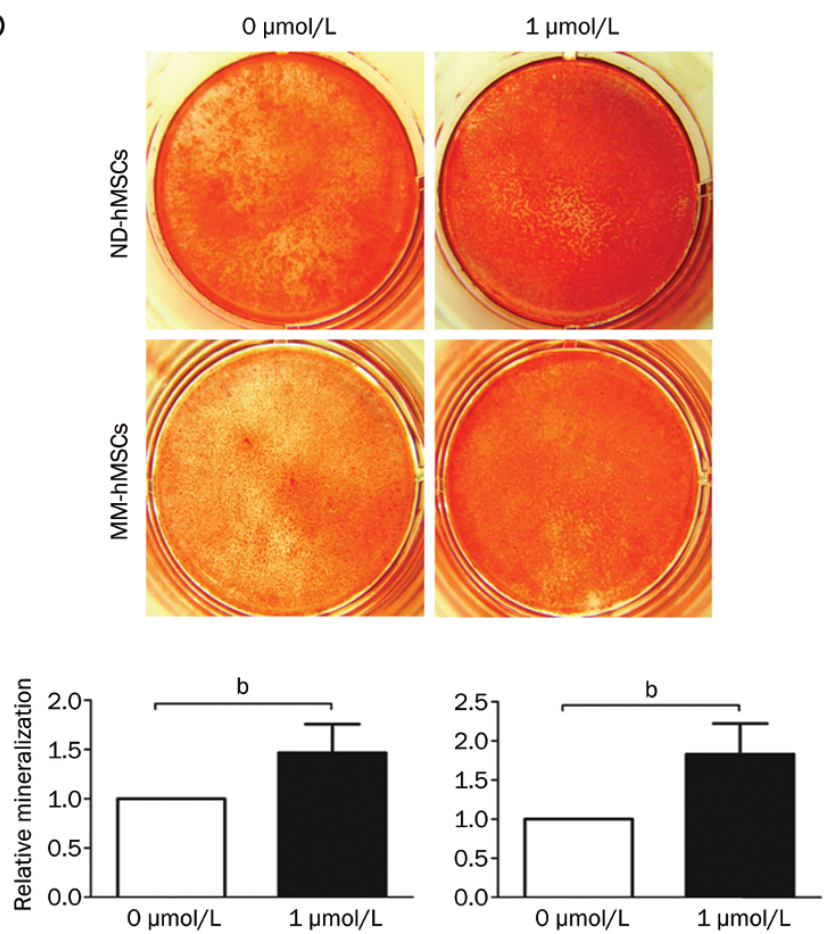

Figure 3. Vorinostat enhances osteogenic differentiation of hMSCs from normal donors and MM patients. (A) ND-hMSCs or MM-hMSCs were incubated for $72 \mathrm{~h}$ in osteogenic medium with different concentrations of vorinostat, and ALP activity was analyzed using an ELISA reader. ${ }^{\mathrm{b}} P<0.05$ compared to the untreated group for ND-hMSCs; ${ }^{e} P<0.05$ compared to the untreated group for MM-hMSCs. (B) ND-hMSCs or MM-hMSCs were cultured for $7 \mathrm{~d}$ in osteogenic medium with or without $1 \mu \mathrm{mol} / \mathrm{L}$ Vorinostat. PCR analyses for OPN, ALP, BSP, BMP2, and $\beta$-actin were performed. A representative PCR result of three independent experiments (upper panels) and densitometric analysis using Image J software (lower panels) are shown; ${ }^{b} P<0.05$. (C) NDhMSCs or MM-hMSCs were cultured for $7 \mathrm{~d}$ in osteogenic medium with or without $1 \mu \mathrm{mol} / \mathrm{L}$ vorinostat. ALP staining was performed using BCIP/NBT solution (Sigma-Aldrich). ALP staining of three independent experiments (upper panels) and quantitative ALP activity using Alkaline Phosphatase Yellow (pNPP) Liquid ELISA substrate (Sigma-Aldrich) (lower panels) are shown; ${ }^{\mathrm{b}} \mathrm{P}<0.05$. (D) ND-hMSCs or MM-hMSCs were cultured in osteogenic medium for $14 \mathrm{~d}$ with or without $1 \mu \mathrm{mol} / \mathrm{L}$ vorinostat. Calcium deposition was demonstrated by Alizarin Red S staining. A representative Alizarin Red S staining of three independent experiments (upper panels) and quantitative mineralization using $10 \%$ cetylpyridinium chloride (lower panels) are shown; Mean \pm SD. ${ }^{\mathrm{b}} \mathrm{P}<0.05$. Abbreviations: OPN, osteopontin; ALP, alkaline phosphatase; BSP, bone sialoprotein; and BMP2, bone morphogenetic protein 2. 
A

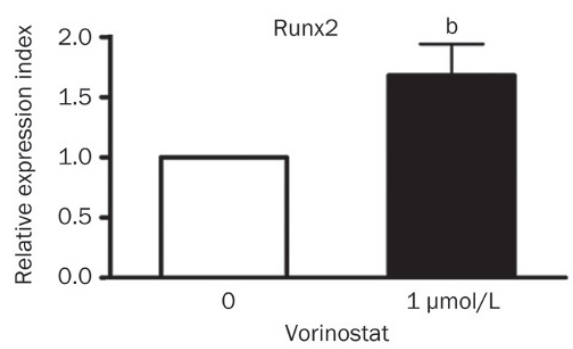

B
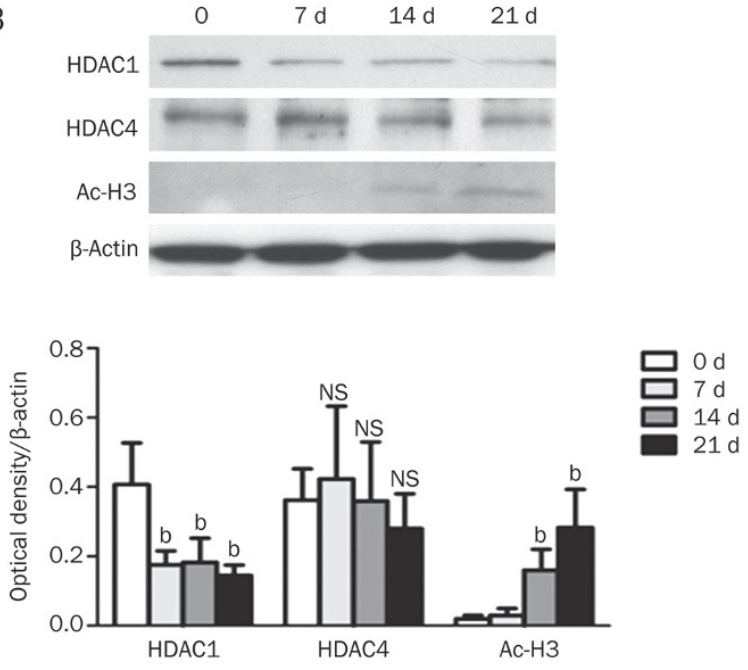

C
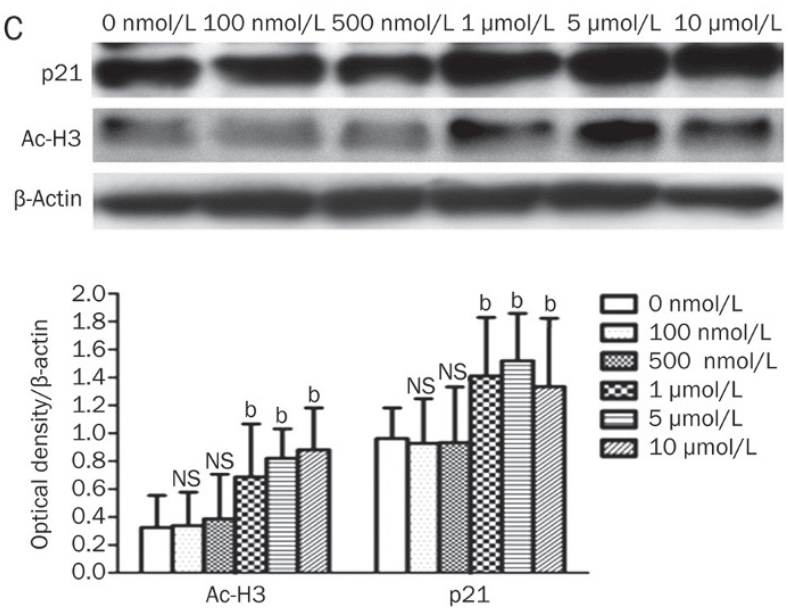

Figure 4. Possible mechanisms for osteogenesis-promoting effect of vorinostat. (A) By real-time PCR, Runx2 expression is upregulated by vorinostat treatment; ${ }^{b} P<0.05$ compared to the untreated group. (B) Western blot analysis of epigenetic alterations during normal hMSC osteogenic differentiation. Representative blots of three independent experiments (upper panels) and densitometric analysis using ImageJ software (lower panels) are shown. NS=not significant; ${ }^{b} P<0.05$ compared to d 0. (C) In the absence of osteogenic stimuli for $48 \mathrm{~h}$, vorinostat induced hyperacetylation of histone $\mathrm{H3}(\mathrm{Ac}-\mathrm{H} 3)$ and increased the expression of $\mathrm{p} 21$. Representative blots of three independent experiments (upper panels) and densitometric analysis using ImageJ software (lower panels) are shown. NS=not significant; ${ }^{\mathrm{b}} \mathrm{P}<0.05$ compared to the untreated group. Mean \pm SD.

A

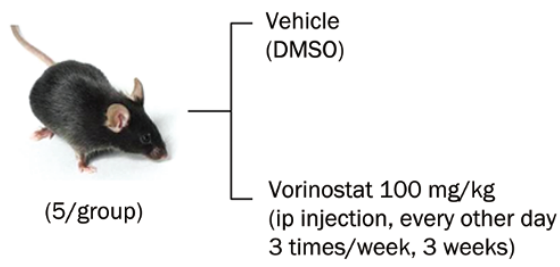

B

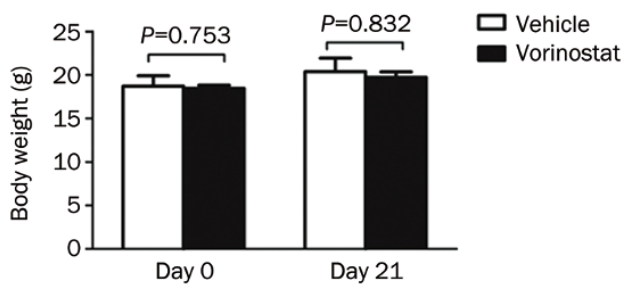

Figure 5. Vorinostat-treated mice did not show a significant decrease in body weight. (A) Treatment schedule. Mice were injected with vehicle (DMSO) or vorinostat ( $100 \mathrm{mg} / \mathrm{kg}$ ) every other day for 3 weeks by ip injection. (B) Body weight was measured on $\mathrm{d} 0$ and $\mathrm{d} 21$. Mean \pm SD.

A

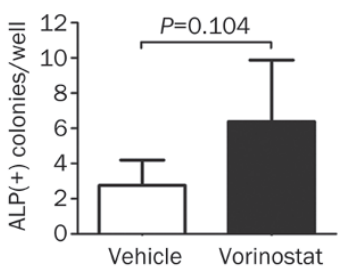

C

\begin{tabular}{lllll}
\hline & \multicolumn{2}{c}{ EC } & \multicolumn{2}{c}{ TRAB } \\
& Ob.N/mm & Oc. $/ \mathrm{mm}$ & Ob.N/mm & Oc. $/ \mathrm{mm}$ \\
\hline Control & $23.90 \pm 2.58$ & $2.21 \pm 0.65$ & $12.39 \pm 2.96$ & $0.93 \pm 0.37$ \\
Vorinostat & $25.51 \pm 2.66$ & $1.56 \pm 0.39$ & $14.81 \pm 4.80$ & $1.06 \pm 0.39$ \\
$P$-value & 0.364 & 0.095 & 0.366 & 0.600 \\
\hline
\end{tabular}

Figure 6. In vivo effect of vorinostat on bone formation in C57BL/KaLwRij mice. (A) Osteogenic colony-forming potential after in vivo treatment with vorinostat. (B) Serum osteocalcin levels were measured by ELISA after 3 weeks of treatment. (C) Histomorphometric analysis of the tibia after vorinostat treatment. Number of osteoblasts $(\mathrm{Ob})$ and osteoclasts $(\mathrm{Oc})$ in the endocortical (EC) bone area and trabecular (TRAB) bone area per $\mathrm{mm}$ were measured. The mean value of two levels in the endocortical bone area and trabecular bone area are shown. $n=5$ per group. Mean \pm SD.

tion, induced by osteogenic stimuli, the expression of HDAC proteins, especially for HDAC1, were gradually downregulated, while the acetylation of histone $\mathrm{H} 3$ increased. It has been proposed that HDAC1 negatively regulates the osteogenic process ${ }^{[23]}$, as the downregulation of HDAC1 induced cellular hyperacetylation, and chromatin structures became more accessible to osteogenic transcription factors, such as 
Runx2. As a pan-inhibitor of class I and class II HDACs, vorinostat inhibits HDAC1 activity, upregulates the cellular acetylation level, and therefore accelerates the natural epigenetic process that occurs during MSC osteogenesis. McGeeLawrence and colleagues reported decreased mineralization and osteoblastic gene expression in murine BM MSCs after vorinostat treatment in vitro ${ }^{[27]}$. However, we noticed that they used primary murine BM nucleated cells in their study but not MSCs. Therefore, this discrepant in vitro data might be related to a difference in the cell population that was studied. Our group and others used in vitro expanded homogeneous human MSCs, whereas McGee-Lawrence et al. tested freshly isolated murine heterogeneous BM cells. In our experience, the majority of primary murine BM cells are hematopoietic cells and not $\mathrm{MSCs}^{[43]}$.

We observed that vorinostat-treated naive mice did not exhibit a decrease in osteogenic colonies, serum osteocalcin, or osteoblast numbers. However, in contrast to our data, two recent studies reported that vorinostat caused bone loss in vivo, as shown by a decrease in the number of osteoblast cells $^{[27,28]}$. We postulate that these discrepant in vivo results are related to the frequency of vorinostat treatment. In the other two studies, mice were treated with vorinostat at $100 \mathrm{mg} / \mathrm{kg}$ ip, daily for 3 or 4 weeks. They observed a significant decrease in body weight, indicating that this treatment schedule causes considerable toxicity in the animals ${ }^{[27]}$. We treated the mice less frequently three times per week), and no significant side effects were observed. Our in vitro data revealed that higher concentrations of vorinostat can affect the viability of MSCs, and therefore, it is crucial to find the optimal concentration of vorinostat that reduces tumor burden without harming the MSC population.

In a phase I trial of single-agent vorinostat in 13 patients with relapsed and/or refractory MM, 1 patient showed a minimal response and 9 patients showed disease stabilization ${ }^{[24]}$. However, because of the early termination of this study due to the sponsor's decision, the maximum tolerated dose (MTD) of vorinostat was not determined, and the activity of vorinostat at an optimum dose and schedule were not fully explored. Campbell RA et al studied the antitumor activity of vorinostat against MM using a LAGK-1B murine xenograft MM model in vivo ${ }^{[31]}$. They reported that vorinostat $(100 \mathrm{mg} / \mathrm{kg})$ daily ip for 2 consecutive days per week markedly decreased the MM tumor burden, and the anti-MM effect of vorinostat was not enhanced when the frequency of drug treatment was increased to 5 consecutive days per week. Collectively, as shown by phase I trial, frequent treatment with vorinostat has a modest single-agent activity in relapsed/refractory MM, and an animal study demonstrated that the anti-MM effect of vorinostat administered at a lower frequency was not significantly different from that of the higher treatment frequency. Because of the risk of inducing bone loss following a high frequency treatment schedule ${ }^{[27,28]}$, a low frequency treatment schedule for the administration of single-agent vorinostat to MM patients seems to be more appropriate. Moreover, we noticed that in the phase I clinical trials of single vorinostat in MM patients, the maximum administered dose of oral vorinostat was $250 \mathrm{mg}$, twice daily (500 mg total). Because the dose used in patients is much lower compared to the dose used in our animal experiments and the oral intake of the drug is less efficient compared to ip injection, it can be assumed that this drug would not induce bone loss in MM patients, even if a more frequent infusion schedule was used.

It is well-documented that MM cells can suppress MSC osteoblastogenesis via multiple molecules ${ }^{[12-16]}$ and activate osteoclastogenesis. Because vorinostat has a strong anti-myeloma effect, a more potent enhancement of bone formation can be expected in MM diseased mice. Our group has demonstrated that JNJ-26481585, another second-generation HDACi, significantly reduced the development of bone disease and tumor burden in the 5T2 MM mouse model ${ }^{[44]}$. Therefore, it would be worth testing the effect of vorinostat in the same model.

Taken together, we could not find any evidence that treatment with vorinostat induces bone loss in an in vivo mouse model. Therefore, we propose that HDACi might be safe to be used as a single agent or in combination with other therapies to target MM cells, without impairing bone formation or eliciting a supportive effect on osteogenesis. However, our findings need to be corroborated with further clinical observation and analysis in MM patients.

\section{Acknowledgements}

We would like to thank Nicole ARRAS, Wim RENMANS, Angelo WILLEMS, and Carine SEYNAEVE for their expert technical assistance and Prof Qing-hua ZHOU for his administrative support. We thank Merck \& Co for providing us with vorinostat. Our research was supported by grants from the FWO-Vlaanderen, "Vlaamse Liga tegen Kanker" (Stichting Emmanuel Van der Schueren), the Vrije Universiteit Brussel (HOA) and the Scientific Foundation Willy Gepts (WFWG) UZ Brussel. Song XU is supported by the CSC-VUB scholarship.

\section{Author contribution}

Song XU and Kim DE VEIRMAN designed the study, performed the experiments, data analysis and interpretation, manuscript writing; Holly EVAMS and Gaia Cecilia SANTINI performed some experiments, data analysis and interpretation; Isabelle VANDE BROEK, Xavier LELEU, and Ann DE BECKER provided study samples; Peter CROUCHER, Ben VAN CAMP, and Karin VANDERKERKEN provided experimental tools and revision of the manuscript; Ivan VAN RIET designed the study and final approval of the manuscript.

\section{References}

1 Kyle RA, Gertz MA, Witzig TE, Lust JA, Lacy MQ, Dispenzieri A, et al. Review of 1027 patients with newly diagnosed multiple myeloma. Mayo Clin Proc 2003; 78: 21-33.

2 Fowler JA, Edwards CM, Croucher PI. Tumor-host cell interactions in the bone disease of myeloma. Bone 2011; 48: 121-8.

3 Bataille R, Chappard D, Marcelli C, Dessauw P, Baldet P, Sany J, et al. Recruitment of new osteoblasts and osteoclasts is the earliest critical 
event in the pathogenesis of human multiple myeloma. J Clin Invest 1991; 88: 62-6.

4 Dominici M, Le Blanc K, Mueller I, Slaper-Cortenbach I, Marini F, Krause D, et al. Minimal criteria for defining multipotent mesenchymal stromal cells. The International Society for Cellular Therapy position statement. Cytotherapy 2006; 8: 315-7.

5 Dazzi F, Ramasamy R, Glennie S, Jones SP, Roberts I. The role of mesenchymal stem cells in haemopoiesis. Blood Rev 2006; 20 : 161-71.

6 Ringdén 0 , Uzunel M, Rasmusson I, Remberger M, Sundberg B, Lönnies $\mathrm{H}$, et al. Mesenchymal stem cells for treatment of therapyresistant graft-versus-host disease. Transplantation 2006; 81: 1390-7.

7 Wakitani S, Mitsuoka T, Nakamura N, Toritsuka Y, Nakamura Y, Horibe S. Autologous bone marrow stromal cell transplantation for repair of full-thickness articular cartilage defects in human patellae: Two case reports. Cell Transplant 2004; 13: 595-600.

8 Kim SM, Lim JY, Park SI, Jeong CH, Oh JH, Jeong M, et al. Gene therapy using TRAIL-secreting human umbilical cord blood-derived mesenchymal stem cells against intracranial glioma. Cancer Res 2008; 68: 9614-23.

9 Corre J, Mahtouk K, Attal M, Gadelorge M, Huynh A, FleuryCappellesso $\mathrm{S}$, et al. Bone marrow mesenchymal stem cells are abnormal in multiple myeloma. Leukemia 2007; 21: 1079-88.

10 Garayoa M, Garcial JL, Santamaria C, Garcia-Gomez A, Blanco JF, Pandiella A. Mesenchymal stem cells from multiple myeloma patients display distinct genomic profile as compared with those from normal donors. Leukemia 2009: 23: 1515-27.

11 Xu S, Evans H, Buckle C, De Veirman K, Hu J, Xu D, et al. Impaired osteogenic differentiation of mesenchymal stem cells derived from multiple myeloma patients is associated with a blockade in the deactivation of the Notch signaling pathway. Leukemia 2012; 26 : 2546-9.

12 Tian E, Zhan F, Walker R, Rasmussen E, Ma Y, Barlogie B, et al. The role of the Wnt-signaling antagonist DKK1 in the development of osteolytic lesions in multiple myeloma. N Engl J Med 2003; 349: 2483-94.

13 Oshima T, Abe M, Asano J, Hara T, Kitazoe K, Sekimoto E, et al. Myeloma cells suppress bone formation by secreting a soluble Wnt inhibitor, sFRP-2. Blood 2005; 106: 3160-5.

14 Standal T, Abildgaard N, Fagerli UM, Stordal B, Hjertner O, Borset M, et al. HGF inhibits BMP induced osteoblastogenesis: possible implications for the bone disease of multiple myeloma. Blood 2007; 109: 3024-30.

15 Ehrlich LA, Chung HY, Ghobrial I, Choi SJ, Morandi F, Colla S, et al. $\mathrm{IL}-3$ is a potential inhibitor of osteoblast differentiation in multiple myeloma. Blood 2005; 106: 1407-14.

16 Vallet S, Pozzi S, Patel K, Vaghela N, Fulciniti MT, Veiby P, et al. A novel role for CCL3 (MIP-1 $\alpha$ ) in myeloma-induced bone disease via osteocalcin downregulation and inhibition of osteoblast function. Leukemia 2011; 25: 1174-81.

17 Giuliani N, Morandi F, Tagliaferri S, Lazzaretti M, Bonomini S, Crugnola $\mathrm{M}$, et al. The proteasome inhibitor bortezomib affects osteoblast differentiation in vitro and in vivo in multiple myeloma patients. Blood 2007; 110: 334-8.

18 Mukherjee S, Raje N, Schoonmaker JA, Liu JC, Hideshima T, Wein MN, et al. Pharmacologic targeting of a stem/progenitor population in vivo is associated with enhanced bone regeneration in mice. J Clin Invest 2008; 118: 491-504.

19 Lee S, Park JR, Seo MS, Roh KH, Park SB, Hwang JW, et al. Histone deacetylase inhibitors decrease proliferation potential and multilineage differentiation capability of human mesenchymal stem cells. Cell Prolif 2009; 42: 711-20.

20 Schroeder TM, Westendorf JJ. Histone deacetylase inhibitors promote osteoblast maturation. J Bone Miner Res 2005; 20: 2254-63.

21 de Boer J, Licht R, Bongers M, van der Klundert T, Arends R, van Blitterswijk C. Inhibition of histone acetylation as a tool in bone tissue engineering. Tissue Eng 2006; 12: 2927-37.

22 Cho HH, Park HT, Kim YJ, Bae YC, Suh KT, Jung JS. Induction of osteogenic differentiation of human mesenchymal stem cells by histone deacetylase inhibitors. J Cell Biochem 2005; 96: 533-42.

23 Lee HW, Suh JH, Kim AY, Lee YS, Park SY, Kim JB. Histone deacetylase 1-mediated histone modification regulates osteoblast differentiation. Mol Endocrinol 2006; 20: 2432-43.

24 Richardson P, Mitsiades C, Colson K, Reilly E, McBride L, Chiao J, et al. Phase I trial of oral vorinostat (suberoylanilide hydroxamic acid, SAHA) in patients with advanced multiple myeloma. Leuk Lymphoma 2008; 49: 502-7.

25 Badros A, Burger AM, Philip S, Niesvizky R, Kolla SS, Goloubeva O, et al. Phase I study of vorinostat in combination with bortezomib for relapsed and refractory multiple myeloma. Clin Cancer Res 2009; 15 : 5250-7.

26 http://clinicaltrials.gov/ct2/show/NCT01554852?term=vorinostat+m yeloma+phase\&rank=20\&submit_fld_opt $=$

27 McGee-Lawrence ME, McCleary-Wheeler AL, Secreto FJ, Razidlo DF, Zhang M, Stensgard BA, et al. Suberoylanilide hydroxamic acid (SAHA; vorinostat) causes bone loss by inhibiting immature osteoblasts. Bone 2011; 48: 1117-26.

28 Pratap J, Akech J, Wixted JJ, Szabo G, Hussain S, McGee-Lawrence $\mathrm{ME}$, et al. The histone deacetylase inhibitor, vorinostat, reduces tumor growth at the metastatic bone site and associated osteolysis, but promotes normal bone loss. Mol Cancer Ther 2012; 9: 3210-20.

29 De Becker A, Van Hummelen P, Bakkus M, Vande Broek I, De Wever J, De Waele $M$, et al. Migration of culture-expanded human mesenchymal stem cells through bone marrow endothelium is regulated by matrix metalloproteinase- 2 and tissue inhibitor of metalloproteinase-3. Haematologica 2007; 92: 440-9.

30 Menu E, Kooijman R, Van Valckenborgh E, Asosingh K, Bakkus M, Van Camp B, et al. Specific roles for the PI3K and the MEK-ERK pathway in IGF-1-stimulated chemotaxis, VEGF secretion and proliferation of multiple myeloma cells: study in the 5T33MM model. Br J Cancer 2004; 90: 1076-83.

31 Campbell RA, Sanchez E, Steinberg J, Shalitin D, Li ZW, Chen H, et al. Vorinostat enhances the antimyeloma effects of melphalan and bortezomib. Eur J Haematol 2010; 84: 201-11.

$32 \mathrm{McGee-Lawrence} \mathrm{ME,} \mathrm{Westendorf} \mathrm{JJ.} \mathrm{Histone} \mathrm{deacetylases} \mathrm{in} \mathrm{skeletal}$ development and bone mass maintenance. Gene 2011; 474: 1-11.

33 Kang JS, Alliston T, Delston R, Derynck R. Repression of Runx2 function by TGF-beta through recruitment of class II histone deacetylases by Smad3. EMBO J 2005; 24: 2543-55.

34 Westendorf JJ, Zaidi SK, Cascino JE, Kahler R, van Wijnen AJ, Lian JB, et al. Runx2 (Cbfa1, AML-3) interacts with histone deacetylase 6 and represses the $\mathrm{p} 21^{\mathrm{CIP1} / \text { WAF1 }}$ promoter. Mol Cell Biol 2002; 22: 7982-92.

35 Schroeder TM, Kahler RA, Li X, Westendorf JJ. Histone deacetylase 3 interacts with Runx2 to repress the osteocalcin promoter and regulate osteoblast differentiation. J Biol Chem 2004; 279: 41998-4200.

36 Haberland M, Mokalled MH, Montgomery RL, Olson EN. Epigenetic control of skull morphogenesis by histone deacetylase 8. Genes Dev 2009; 23: 1625-30.

37 Bäckesjö CM, Li Y, Lindgren U, Haldosén LA. Activation of Sirt1 decreases adipocyte formation during osteoblast differentiation of mesenchymal stem cells. J Bone Miner Res 2006; 21: 993-1002. 
38 Finnin MS, Donigian JR, Cohen A, Richon VM, Rifkind RA, Marks PA, et al. Structures of a histone deacetylase homologue bound to the TSA and SAHA inhibitors. Nature 1999; 401: 188-93.

39 Kumagai T, Wakimoto N, Yin D, Gery S, Kawamata N, Takai N, et al. Histone deacetylase inhibitor, suberoylanilide hydroxamic acid (vorinostat, SAHA) profoundly inhibits the growth of human pancreatic cancer cells. Int J Cancer 2007; 121: 656-65.

40 Galanis E, Jaeckle KA, Maurer MJ, Reid JM, Ames MM, Hardwick JS, et al. Phase II trial of vorinostat in recurrent glioblastoma multiforme: a north central cancer treatment group study. J Clin Oncol 2009; 27: 2052-8.

41 Di Bernardo G, Squillaro T, Dell'Aversana C, Miceli M, Cipollaro M, Cascino A, et al. Histone deacetylase inhibitors promote apoptosis and senescence in human mesenchymal stem cells. Stem Cells Dev
2009; 18: 573-81.

42 Meng J, Zhang HH, Zhou CX, Li C, Zhang F, Mei QB. The histone deacetylase inhibitor trichostatin $A$ induces cell cycle arrest and apoptosis in colorectal cancer cells via p53-dependent and -independent pathways. Oncol Rep 2012; 28: 384-8.

43 Xu S, De Becker A, Van Camp B, Vanderkerken K, Van Riet I. An improved harvest and in vitro expansion protocol for murine bone marrow-derived mesenchymal stem cells. J Biomed Biotechnol 2010; 2010: 105940.

44 Deleu S, Lemaire M, Arts J, Menu E, Van Valckenborgh E, King P, et al. The effects of JNJ-26481585, a novel hydroxamate-based histone deacetylase inhibitor, on the development of multiple myeloma in the 5T2MM and 5T33MM murine models. Leukemia 2009; 23: 1894903. 